\section{Comparison of Ketamine Alone and Subanesthetic Dose of Ketamine-Fentanyl for Regional Anesthesia in Children}

Sir,

There are major differences between children and adults in anatomical structure of body, physiological function of organs, utilisation and excretion of therapeutic agents, and so on. Therefore, pediatric anesthesia is different from adult anesthesia. Regional anesthesia is usually used in anesthesia for operations of abdomen and lower limbs in children in order to reduce adverse effects of anesthetic drugs on central nervous system and brain function in children.Because of the small age and poor compliance of children, regional anesthesia for children continues to grow in popularity. Ketamine is the only general anesthesia drug with sedative and analgesic effects. Ketamine produces dissociative anaesthesia. There is no other drug to replace ketamine for pediatric anesthesia at present. ${ }^{1}$ In traditional ketamine anesthesia alone, although the anesthetic effect is good, but the dose of ketamine is relatively large, which often leads adverse reactions such as dyspnea, restlessness and mental disorders, and then affects the prognosis of children.

In order to reduce the dosage of ketamine, people actively explore the feasibility of ketamine combinations. As a synthetic opioid derivative, fentanyl has good analgesic and anesthetic effects. Fentanyl has the advantages of fast acting and mild side effects in pediatric surgery anesthesia. ${ }^{2} \mathrm{~A}$ study has shown that ketamine combined with fentanyl can improve pain control after tonsillectomy in children. ${ }^{3}$ The author also found that subanesthetic dose of ketamine-fentanyl assisted regional anesthesia for children, who underwent abdominal or limb surgery, could also achieve good anesthetic effects.

A total of 82 children, who underwent abdominal or limb surgery, were selected as the study subjects. Children with nervous system diseases, blood coagulation dysfunctions and combined with major organ dysfunctions were excluded. All children were randomly divided into group A and group B with 41 cases in each group.

All children were subjected to fasting for six hours before operation, and water deprivation 4 hours before operation. Half an hour before operation, $3 \mathrm{mg} / \mathrm{kg}$ phenobarbital sodium and $0.02 \mathrm{mg} / \mathrm{kg}$ atropine were injected intramuscularly to reduce gland secretion, and $5 \mathrm{mg} / \mathrm{kg}$ ketamine was injected intramuscularly after entering the operating room for basic anesthesia. In group A, ketamine anesthesia alone was used.

Subanaesthetic dose of ketamine-fentanyl anesthesia was used in group B, namely, $1.5 \mathrm{mg} / \mathrm{kg}$ fentanyl and $0.25 \mathrm{mg} / \mathrm{kg}$ ketamine were injected intravenously and spinal canal block with $25 \mathrm{G}$ lumbar puncture needle was used, and then $3 \mathrm{~mL} 1.5 \%$ lidocaine was injected after the success of catheterisation puncture. Operation was performed after the success of regional anesthesia. The anesthetic effects of the two groups were compared. Data was analysed using SPSS version 21. Independent sample t-test and Chi-square test were used for statistical comparisons of quantitative and qualitative variables, respectively. The $p$-value $<0.05$ was considered significant.

Among the 82 children, 43 cases were males (52.44\%) and 39 cases were females $(47.56 \%)$. The age ranged from 3 to $10(5.73 \pm 1.05)$ years. Forty-eight cases were in grade I $(58.54 \%)$, and 34 cases were in grade II (41.46\%), according to ASA classification. Thirty-one cases $(37.80 \%)$ underwent abdominal surgery, and 51 cases $(62.20 \%)$ underwent limb surgery.

The total dosage of ketamine in group B was 52.64 $\pm 6.85 \mathrm{mg} / \mathrm{kg}$, which was lower than $135.72 \pm 15.33 \mathrm{mg} / \mathrm{kg}$ of group A ( $p<0.001)$. The wake-up time of group B was $6.42 \pm 1.05 \mathrm{~min}$, which was shorter than $29.75 \pm 10.31$ min of group A $(p<0.001)$.

The frequency of adverse reactions in group $A$ was $19.51 \%$ (8 cases), including vomiting in four cases $(9.76 \%)$, restlessness in three cases $(7.32 \%)$, and respiratory depression in one case $(2.44 \%)$. The frequency of adverse reactions in group B was $4.88 \%$ ( 2 cases), including vomiting and restlessness in one case $(2.44 \%)$ each. The incidence of adverse reactions in group $A$ was lower than that in group $B(p=0.043)$.

A meta-analysis showed that there was no evidence that any of the three substances (ketamine, fentanyl and morphine) was superior to other drugs. ${ }^{4}$ Akelma et al. have confirmed that compared with ketamine-midazolam combinations, ketamine-fentanyl combinations had better hemodynamic characteristics and fewer side effects in extracorporeal shock wave lithotripsy in children. ${ }^{5}$

The results showed that subanesthetic dose of ketamine-fentanyl had better clinical effects in regional anesthesia for children than ketamine alone, and the dosage of ketamine and the incidence of adverse reactions were less. The reason may be that the combination of fentanyl and low dose of ketamine not only have synergistic effect in anesthesia, but can more effectively maintain the stability of vital signs of patients, 
and to some extent offset the adverse reactions between them. The anesthesia method of subanaesthetic dose of ketamine-fentanyl is worthy of clinical application.

\section{REFERENCES}

1. Bergman SA. Ketamine: Review of its pharmacology and its use in pediatric anesthesia. Anesth Prog 1999; 46:10-20.

2. Taheri R, Seyedhejazi M, Ghojazadeh M, Ghabili K, Shayeghi S. Comparison of ketamine and fentanyl for postoperative pain relief in children following adenotonsillectomy. Pak J Biol Sci 2011; 14:572-7.

3. Elshammaa N, Chidambaran V, Housny W, Thomas J, Zhang X, Michael R. Ketamine as an adjunct to fentanyl improves postoperative analgesia and hastens discharge in children following tonsillectomy - a prospective, double-blinded, randomized study. Paediatr Anaesth 2011; 21:1009-14.

4. Häske D, Böttiger BW, Bouillon B, Fischer M, Gaier G, Gliwitzky $B$, et al. Analgesia in patients with trauma in emergency medicine. Dtsch Arztebl Int 2017; 114:785-92.

5. Akelma H, Kiliç ET, Salik F, Kaydu A. Comparison of ketamine with midazolam versus ketamine with fentanyl for pediatric extracorporeal shock wave lithotripsy procedure: A randomized controlled study. Anesth Essays Res 2018; 12:464-9.

\section{CONFLICT OF INTEREST:}

Authors declared no conflict of interest.

\section{AUTHORS' CONTRIBUTION:}

LZ: Drafted the work, the conception or design of the work; the acquisition, analysis, or interpretation of data for the work.

HL: Drafted the work or revising it critically for important intellectual content.

BZ: Revised manuscript critically for important intellectual content, final approval of the version to be published.

Liang Zhongl, Hongtu $\mathrm{Li}^{2}$ and Bange Zhao ${ }^{3}$

1 Department of Anesthesiology, Wuhan Children's Hospital (Wuhan Maternal and Child Healthcare Hospital), Tongji

Medical College, Huazhong University of Science \& Technology, 430016, China

2 Department of Synthetic Painless Endoscopy, Linzi District People's Hospital, 255400, China

3 Department of Anesthesiology, Zhoupu Hospital of Pudong New District Shanghai 201318, China

Correspondence to: Bange Zhao, Department of Anesthesiology, Zhoupu Hospital of Pudong, New District Shanghai 201318, China

E-mail:lte254@163.com

Received: December 07, 2018; Revised: April 16, 2019; Accepted: April 22, 2019. 